\title{
Photo- and Electron-Production of Mesons on Nucleons and Nuclei
}

\author{
E. Oset ${ }^{1}$, M. Döring ${ }^{1}$, D. Strottman ${ }^{1}$, D. Jido ${ }^{2}$, M. Napsuciale ${ }^{1}$, K. Sasaki ${ }^{1}$, \\ C. A. Vaquera-Araujo ${ }^{1}$, M. Kaskulov ${ }^{3}$, E. Hernandez ${ }^{4}$, H. Nagahiro ${ }^{5}$, S. Hirenzaki ${ }^{6}$ \\ ${ }^{1}$ Dep. de Fisica Teorica and IFIC, Centro Mixto Universidad de Valencia \\ CSIC, Institutos de Investigación de Paterna, Aptd. 22085, 46071 Valencia, Spain \\ ${ }^{2}$ Yukawa Institute for Theoretical Physics, Kyoto University, Kyoto, 606-8502, Japan \\ ${ }^{3}$ Institut für Theoretische Physik, Universität Giessen, Germany \\ ${ }^{4}$ Facultad de Ciencias, Universidad de Salamanca, E-37008 Salamanca, Spain \\ ${ }^{5}$ Research Center for Nuclear Physics, Osaka University \\ Ibaraki, Osaka 567-0047 Japan \\ ${ }^{6}$ Department of Physics, Nara Women's University, Nara 630-8506 Japan
}

August 16, 2021

\begin{abstract}
In these lectures I will show some results obtained with the chiral unitary approach applied to the photo and electroproduction of mesons. The results for photoproduction of $\eta \pi^{0} p$ and $K^{0} \pi^{0} \Sigma^{+}$, together with related reactions will be shown, having with common denominator the excitation of the $\Delta(1700)$ resonance which is one of those dynamically generated in the chiral unitary approach. Then I will show results obtained for the $e^{+} e^{-} \rightarrow \phi f_{0}(980)$ reaction which reproduce the bulk of the data except for a pronounced peak, giving support to a new mesonic resonance, $\mathrm{X}(2175)$. Results will also be shown for the electromagnetic form factors of the $N^{*}(1535)$ resonance, also dynamically generated in this approach. Finally, I will show some results on the photoproduction of the $\omega$ in nuclei, showing that present experimental results claiming a shift of the $\omega$ mass in the medium are tied to a particular choice of background and are not conclusive. On the other hand, the same experimental results show unambiguously a huge increase of the $\omega$ width in the nuclear medium.
\end{abstract}

\section{Introduction}

Nowadays it is commonly accepted that QCD is the theory of the strong interactions, with the quarks as building blocks for baryons and mesons, and the gluons as the mediators of the interaction. However, at low energies typical of the nuclear phenomena, perturbative calculations with the QCD Lagrangian are not possible and one has to resort to other techniques to use the information of the QCD Lagrangian. One of the most fruitful approaches has been the use of chiral perturbation theory, $\chi P T$ [1, 2, 3]. The theory introduces effective Lagrangians which involve only observable particles, mesons and baryons, 
respects the basic symmetries of the original QCD Lagrangian, particularly chiral symmetry, and organizes these effective Lagrangians according to the number of derivatives of the meson and baryon fields.

The introduction of unitarity constraints in coupled channels in chiral perturbation theory has led to unitary extensions of the theory that starting from the same effective Lagrangians allow one to make predictions at much higher energies. One of the interesting consequences of these extensions is that they generate dynamically low lying resonances, both in the mesonic and baryonic sectors. By this we mean that they are generated by the multiple scattering of the meson or baryon components, much the same as the deuteron is generated by the interaction of the nucleons through the action of a potential, and they are not preexistent states that remain in the large $N_{c}$ limit where the multiple scattering is suppressed.

\section{Baryon meson interaction}

The interaction of the octet of pseudoscalar mesons with the octet of stable baryons has been the object of much theoretical study [4, 5, 6, 17, 9, 10, 11, 12, 13, 14, 15, 16, 17, 18. One of the common features of these studies is the dynamical generation of some resonances which appear as a consequence of the interaction of the mesons and baryons in coupled channels, forming some kind of molecular state, quite different for the ordinary three quark states associated to the baryons. The $\Lambda(1405), N *(1535), \Lambda(1670)$, etc, are examples of these states. I will not expose here the formalism which can be found in all these references ( a lecture type approach to the problem can bee seen in [19]). Instead, I will briefly expose the formalism for the interaction of the octet of pseudoscalar mesons with the decuplet of baryons, which has received far less attention [20, 21, 14]. In this approach one of the resonances that appears dynamically generated is the $\Delta(1700)$ which will play an important role in some of the reactions which I report here.

The lowest order term of the chiral Lagrangian relevant for the interaction of the baryon decuplet with the octet of pseudoscalar mesons is given by [22]

$$
\mathcal{L}=-i \bar{T}^{\mu} \mathcal{D} T_{\mu}
$$

where $T_{a b c}^{\mu}$ is the spin decuplet field and $D^{\nu}$ the covariant derivative given by

$$
\mathcal{D}^{\nu} T_{a b c}^{\mu}=\partial^{\nu} T_{a b c}^{\mu}+\left(\Gamma^{\nu}\right)_{a}^{d} T_{d b c}^{\mu}+\left(\Gamma^{\nu}\right)_{b}^{d} T_{a d c}^{\mu}+\left(\Gamma^{\nu}\right)_{c}^{d} T_{a b d}^{\mu}
$$

where $\mu$ is the Lorentz index, $a, b, c$ are the $S U(3)$ indices. The vector current $\Gamma^{\nu}$ is given by

$$
\Gamma^{\nu}=\frac{1}{2}\left(\xi \partial^{\nu} \xi^{\dagger}+\xi^{\dagger} \partial^{\nu} \xi\right)
$$

with

$$
\xi^{2}=U=e^{i \sqrt{2} \Phi / f}
$$

where $\Phi$ is the ordinary $3 \times 3$ matrix of fields for the pseudoscalar mesons [1] and $f=93 \mathrm{MeV}$. We deal here only about the $S$-wave part of the baryon meson interaction and take for the Rarita-Schwinger fields $T_{\mu}$ the representation $T u_{\mu}$ from ref. [23, 24] with $u_{\mu}$ the Rarita-Schwinger spinor.

Let us recall the identification of the $S U(3)$ component of $T$ to the physical states [25, 26]:

$T^{111}=\Delta^{++}, T^{112}=\frac{1}{\sqrt{3}} \Delta^{+}, T^{122}=\frac{1}{\sqrt{3}} \Delta^{0}, T^{222}=\Delta^{-}, T^{113}=\frac{1}{\sqrt{3}} \Sigma^{*+}, T^{123}=\frac{1}{\sqrt{6}} \Sigma^{* 0}, T^{223}=\frac{1}{\sqrt{3}} \Sigma^{*-}$, $T^{133}=\frac{1}{\sqrt{3}} \Xi^{* 0}, T^{233}=\frac{1}{\sqrt{3}} \Xi^{*-}, T^{333}=\Omega^{-}$.

\footnotetext{
${ }^{1}$ We use the metric $g_{\mu \nu}=\operatorname{diag}(1,-1,-1,-1)$.
} 
For a meson of incoming (outgoing) momenta $k\left(k^{\prime}\right)$ one obtains the simple form for the $S$-wave transition amplitudes, similar to [6],

$$
V_{i j}=-\frac{1}{4 f^{2}} C_{i j}\left(k^{0}+k^{\prime 0}\right)
$$

The coefficients $C_{i j}$ for reactions with all possible values of strangeness $(S)$ and charge $(Q)$ are given in [21]. This interaction plays the role of the kernel in the Bethe Salpeter equation which is used to construct the scattering t-matrix between the different channels. In the next section we approach this point.

\section{Unitarized chiral perturbation theory: N/D or dispersion relation method}

One can find a systematic and easily comprehensible derivation of the ideas of the N/D method applied for the first time to the meson baryon system in [7] in the context of chiral dynamics, which we reproduce here below and which follows closely the similar developments used before in the meson meson interaction [8]. One defines the transition $T$-matrix as $T_{i, j}$ between the coupled channels which couple to certain quantum numbers. For instance in the case of $\bar{K} N$ scattering studied in [7] the channels with zero charge are $K^{-} p, \bar{K}^{0} n, \pi^{0} \Sigma^{0}, \pi^{+} \Sigma^{-}, \pi^{-} \Sigma^{+}, \pi^{0} \Lambda, \eta \Lambda, \eta \Sigma^{0}, K^{+} \Xi^{-}, K^{0} \Xi^{0}$. Unitarity in coupled channels is written as

$$
\operatorname{Im} T_{i, j}=T_{i, l} \rho_{l} T_{l, j}^{*}
$$

where $\rho_{i} \equiv 2 M_{l} q_{i} /(8 \pi W)$, with $q_{i}$ the modulus of the c.m. three-momentum, and the subscripts $i$ and $j$ refer to the physical channels. This equation is most efficiently written in terms of the inverse amplitude as

$$
\operatorname{Im} T^{-1}(W)_{i j}=-\rho(W)_{i} \delta_{i j}
$$

The unitarity relation in Eq. (17) gives rise to a cut in the $T$-matrix of partial wave amplitudes, which is usually called the unitarity or right-hand cut. Hence one can write down a dispersion relation for $T^{-1}(W)$

$$
T^{-1}(W)_{i j}=-\delta_{i j}\left\{\widetilde{a}_{i}\left(s_{0}\right)+\frac{s-s_{0}}{\pi} \int_{s_{i}}^{\infty} d s^{\prime} \frac{\rho\left(s^{\prime}\right)_{i}}{\left(s^{\prime}-s\right)\left(s^{\prime}-s_{0}\right)}\right\}+\mathcal{T}^{-1}(W)_{i j},
$$

where $s_{i}$ is the value of the $s$ variable at the threshold of channel $i$ and $\mathcal{T}^{-1}(W)_{i j}$ indicates other contributions coming from local and pole terms, as well as crossed channel dynamics but without righthand cut. These extra terms are taken directly from $\chi P T$ after requiring the matching of the general result to the $\chi P T$ expressions. Notice also that

$$
g(s)_{i}=\widetilde{a}_{i}\left(s_{0}\right)+\frac{s-s_{0}}{\pi} \int_{s_{i}}^{\infty} d s^{\prime} \frac{\rho\left(s^{\prime}\right)_{i}}{\left(s^{\prime}-s\right)\left(s^{\prime}-s_{0}\right)}
$$

is the familiar scalar loop integral.

One can further simplify the notation by employing a matrix formalism. Introducing the matrices $g(s)=\operatorname{diag}\left(g(s)_{i}\right), T$ and $\mathcal{T}$, the latter defined in terms of the matrix elements $T_{i j}$ and $\mathcal{T}_{i j}$, the $T$-matrix can be written as:

$$
T(W)=[I-\mathcal{T}(W) \cdot g(s)]^{-1} \cdot \mathcal{T}(W)
$$

which can be recast in a more familiar form as

$$
T(W)=\mathcal{T}(W)+\mathcal{T}(W) g(s) T(W)
$$


Now imagine one is taking the lowest order chiral amplitude for the kernel $\mathcal{T}$ as done in [7]. Then the former equation is nothing but the Bethe Salpeter equation with the kernel taken from the lowest order Lagrangian and factorized on shell, the same approach followed in [6], where different arguments were used to justify the on shell factorization of the kernel.

The on shell factorization of the kernel, justified here with the N/D method, renders the set of coupled Bethe Salpeter integral equations a simple set of algebraic equations.

In the case of the interaction of the octet of pseudoscalar mesons with the baryon decuplet, one of the resonances which appears dynamically generated is the $\Delta(1700)$, which is built from the coupled channels $\pi \Delta, \eta \Delta$ and $K \Sigma(1535)$. The study of the amplitudes around the pole allows one to get the mass, the width, and through the residues at the pole, the coupling of the resonance to the different channels. Another one of such states is the $\Lambda(1520)$ which couples to the $\pi \Sigma(1385)$ and $K \Xi(1530)$ channels, although it has also an appreciable coupling to the $\pi \Sigma$ and $\bar{K} N$ channels, responsible for its width, and to which it couples in D-waves [27].

\section{Clues to the nature of the $\Delta(1700)$ resonance from pion and photon induced reactions}

As mentioned in the previous section, the $\Delta(1700)$ is a dynamically generated resonance from the $\pi \Sigma$ and $\bar{K} N$ channels with the interaction discussed in section 2 . The couplings to these channels are evaluated in [21]. If we study the $\gamma p \rightarrow \pi^{0} \eta p$ or the $\gamma p \rightarrow \pi^{0} K^{0} \Sigma^{+}$reactions it was found in [28] and [29] that the mechanisms of $\Delta(1700)$ excitation with posterior decay of the $\Delta(1700)$ into $\eta \Delta$ and $K^{0} \Sigma(1385)$ were dominant in these reactions at low energies. The corresponding mechanisms are depicted in fig 1 .
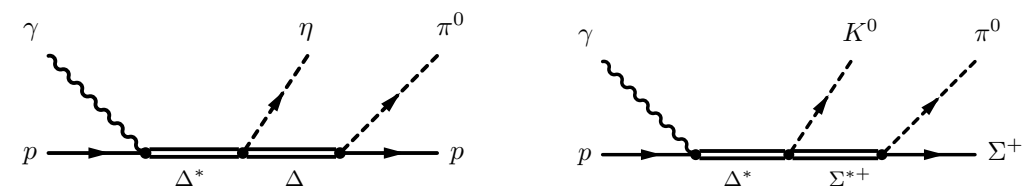

Figure 1: Tree level processes from the decay of the $\Delta^{*}(1700)$ to $\eta \Delta(1232)$ and $K^{0} \Sigma^{*+}$.

By analogy, reactions with the same final state induced by other probes should show similar features. In fig. 2 we show the corresponding Feynman diagrams for pion induced reactions.

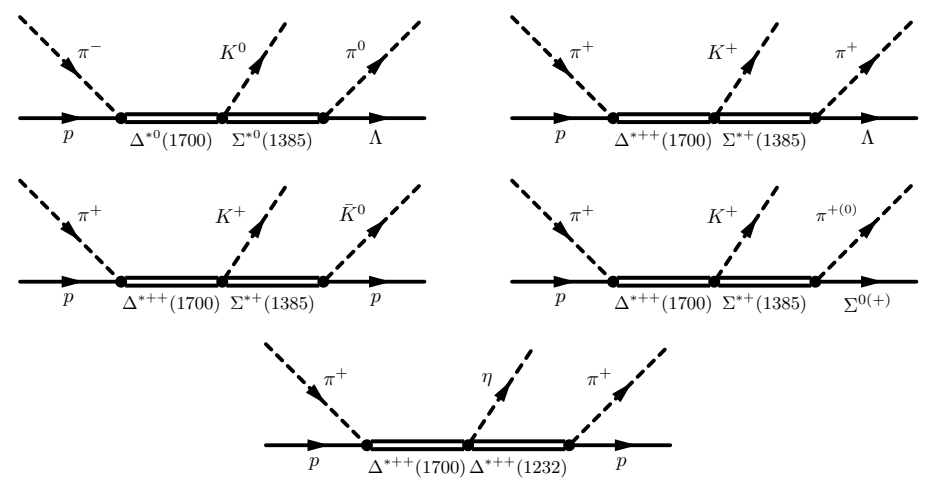

Figure 2: Tree level contributions for the pion-induced strangeness production.

In fig. 3 we show the results for the cross sections for several pion induced reactions which were evaluated in [29]. The overall agreement with the data is fair. However, this fair agreement gains more 
strength after the following considerations. The $\Delta(1700)$ is assumed to be a member of a decuplet in the PDG. If one assumes this, it is easy, using SU(3) Clebsch Gordan coefficients, to see that the couplings to the $\Delta \pi, \Sigma^{*} K, \Delta \eta$ states in $I=3 / 2$ are proportional to $\sqrt{5 / 8}, \sqrt{1 / 4}, \sqrt{1 / 8}$, respectively. The squares of these coefficients are proportional to $1,2 / 5,1 / 5$, respectively, compared to the squares of the coefficients of the dynamically generated resonance, $1,11.56,4.84$. With respect to the decuplet assumption of the PDG one obtains factors 27.5 and 24 larger for the square of the couplings to $\Sigma^{*} K$ and $\Delta \eta$, respectively. In some cross sections involving the square of these numbers the differences would be huge. With this perspective, the fair agreement found with experiment has a more significant value.
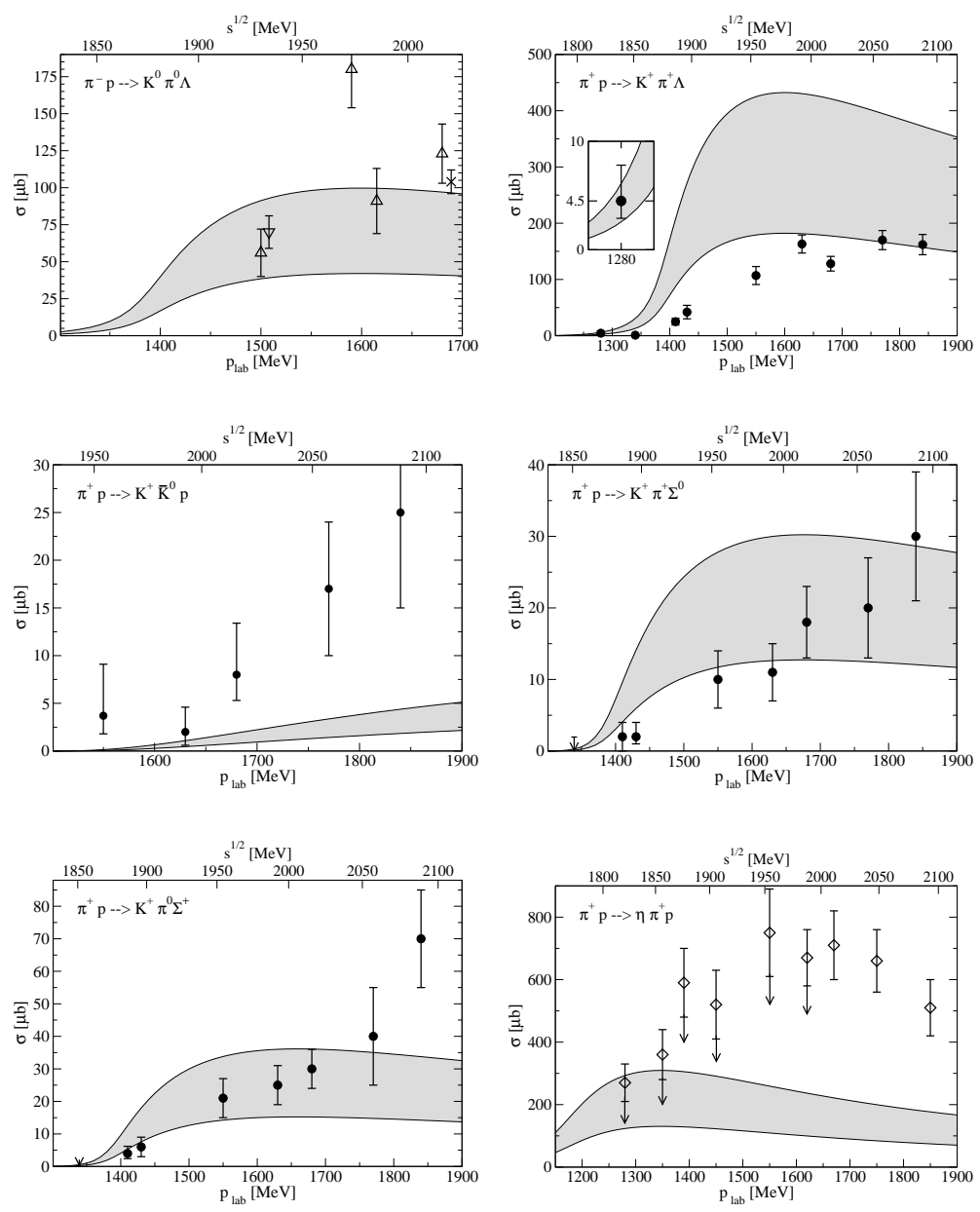

Figure 3: Total cross sections for the pion-induced reactions. Data are from [30] (triangles up), [31] (triangle down), [34] (cross), [32] (dots), 33] (diamonds). For the latter data, it is indicated that these are upper limits below $p=1.67 \mathrm{GeV}$ as in Ref. [33].

As we indicated, the case for $K^{0} \pi^{0} \Lambda$ is dominated in our model by $K^{0} \Sigma(1385)$ production. This is the case experimentally, as one can see in fig. 4.

The case of photoproduction is also instructive. In fig. 5 we can see that there is a good agreement with the data, though the experimental errors are large. The precision of the experiment has been significantly improved in recent preliminary measurements at ELSA, and the agreement of our results with the data is very good [35]. Finally, in the same figure we can see the predictions for the $\gamma p \rightarrow$ $\pi^{0} \eta p$ and $\gamma p \rightarrow K^{0} \pi^{0} \Sigma^{+}$production which have also been measured recently at ELSA [36] and the agreement with the data is also good, as well as with the data from [37] and recent data by the GRAAL collaboration on $\gamma p \rightarrow \pi^{0} \eta p$ [38], where is addition we find also good agreement with the asymmetries [38]. It is worth stating that in the absence of the $\Delta(1700)$ intermediate state (the model of [28] has 

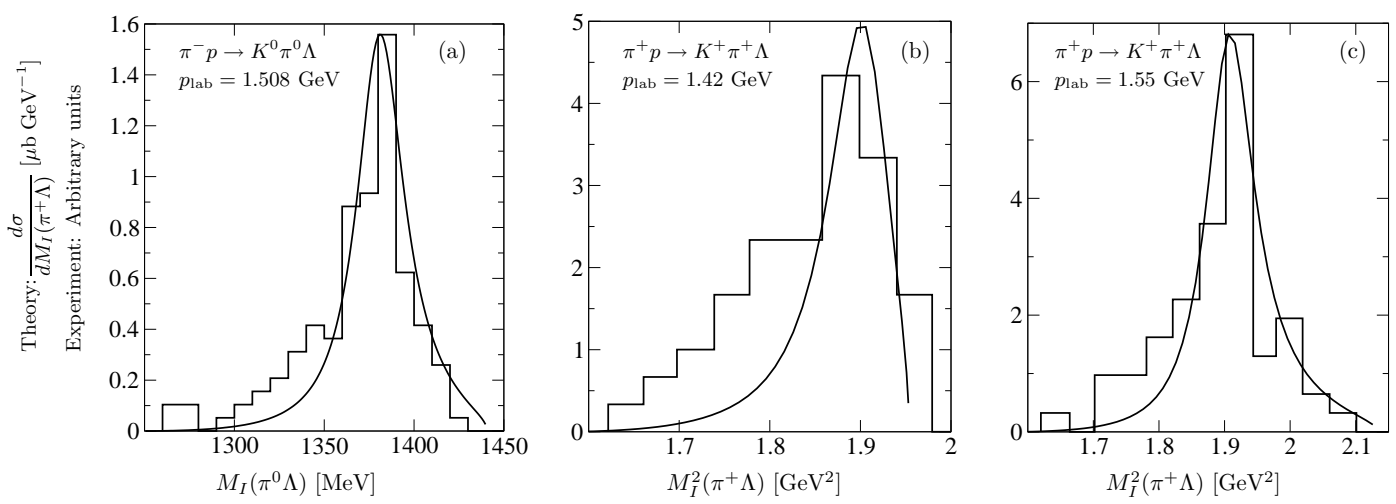

Figure 4: Invariant mass spectra for the $\pi^{-} p \rightarrow K^{0} \pi^{0} \Lambda$ and $\pi^{+} p \rightarrow K^{+} \pi^{+} \Lambda$ reactions. Experimental distributions (arbitrary units) are from [31] and [32], respectively.

more terms than just the $\Delta(1700)$ excitation), the results for the asymmetries are in sheer disagreement with the data.
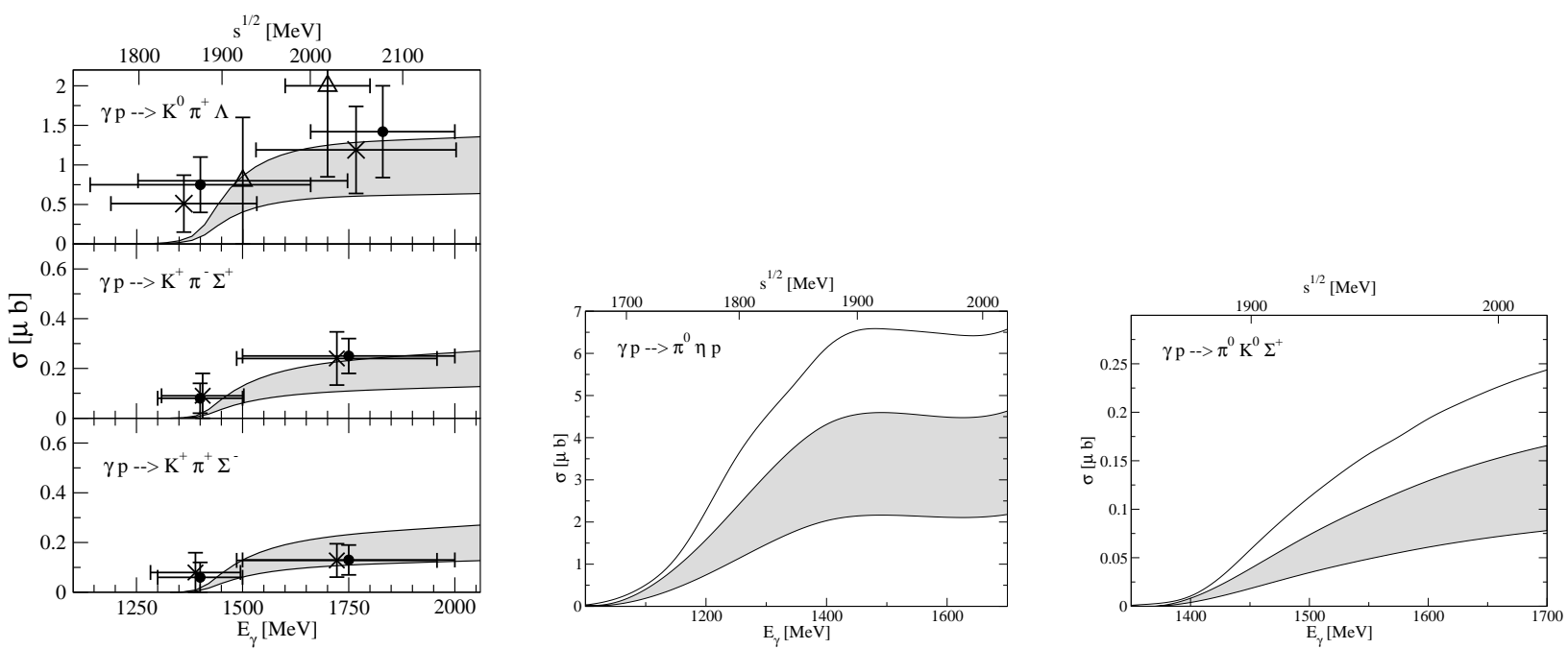

Figure 5: Photoproduction of strange and $\eta$ particles. Data are from [39] (dots), [40] (crosses), and [41] (triangles up). The latter data are the sum of $K \Lambda \pi$ and $K \Sigma \pi$ final states. The two lower plots show the update of the predictions from Ref. [28] (gray bands) compared to the results from [29] (solid lines).

\section{The $e^{+} e^{-} \rightarrow \phi f_{0}(980)$ and clues for a new mesonic resonance $\mathrm{X}(2175)$}

In recent experiments [42, 43] the $e^{+} e^{-} \rightarrow \phi \pi^{+} \pi^{-}$and $e^{+} e^{-} \rightarrow \phi \pi^{0} \pi^{0}$ cross sections were measured and a pronounced peak was found for the invariant mass of the two pions in the region of the $f_{0}(980)$ scalar meson resonance. By looking at the energy dependence of the $e^{+} e^{-} \rightarrow \phi f_{0}(980)$ a neat peak was found on top of a smooth background which was identified in [42, 43] with a new resonance X(2175). We have attempted to reproduce these data by recalling the model used for the $\phi \rightarrow \gamma f_{0}(980)$ in [44] (see also other works in the same direction [45]), which would be the time reversal reaction of the present one, with the novelty of having virtual photons. We have used the Feynman diagrams of fig. 6, which involve kaons as intermediate states. We observed that because one has larger energies than in the case 
of the $\phi$, we had to introduce also $K^{*}$ in the intermediate states. The production of the $f_{0}(980)$ is done automatically in our approach, since this resonance is also dynamically generated by the interaction of meson-meson in coupled channels. Hence, the t-matrices that we implement in the loops automatically generate this resonance which does not have to be introduced by hand. The details can be seen in [46].

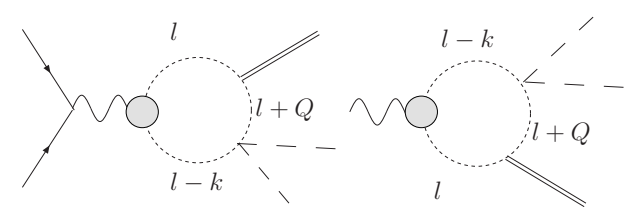

a)

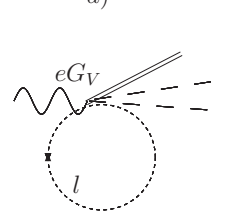

d) b)

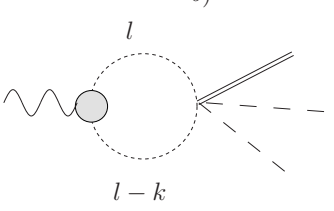

e)

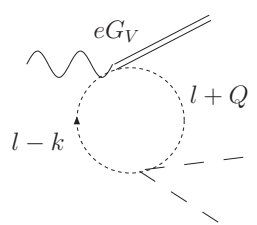

c)

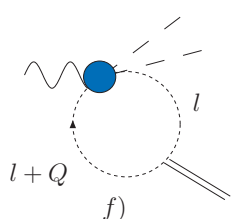

f)

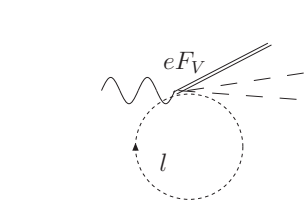

g)
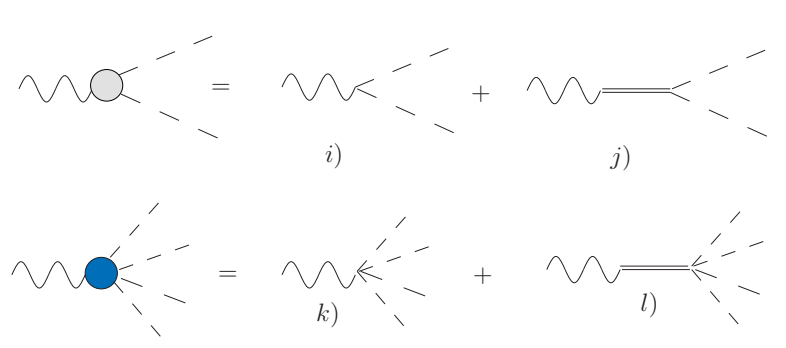

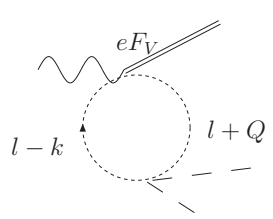

h)

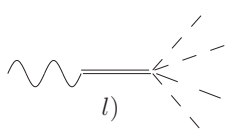

Figure 6: Feynman diagrams for $e^{+} e^{-} \rightarrow \phi \pi \pi$ in $R \chi P T$.

The evaluation of the amplitude requires to work out the loop tensor $T_{\mu \nu}^{a b c}$, such that the amplitude is $T_{\mu \nu}^{a b c} \epsilon_{\mu}(\gamma) \epsilon_{\nu}(\phi)$. It can be shown that $T_{\mu \nu}^{a b c}$ is finite and gauge invariant. The most general form of this tensor is

$$
T_{\mu \nu}^{a b c}=a g_{\mu \nu}+b Q_{\mu} Q_{\nu}+c Q_{\mu} k_{\nu}+d k_{\mu} Q_{\nu}+e k_{\mu} k_{\nu}
$$

where $a, b, c, d, e$ are form factors and $\mathrm{k}$ and $\mathrm{Q}$ are the momenta of the photon and the $\phi$ respectively. Gauge invariance requires

$$
k^{\mu} T_{\mu \nu}^{a b c}=\left(a+c k \cdot Q+e k^{2}\right) k_{\nu}+\left(b k \cdot Q+d k^{2}\right) Q_{\nu}=0,
$$

imposing the following relations among the form factors

$$
a=-c k \cdot Q-e k^{2}, \quad b k \cdot Q=-d k^{2},
$$

thus $T_{\mu \nu}^{a b c}$ has the following explicitly gauge invariant form

$$
T_{\mu \nu}^{a b c}=-c\left(Q \cdot k g_{\mu \nu}-Q_{\mu} k_{\nu}\right)-\frac{d}{k \cdot Q}\left(k^{2} Q_{\mu}-k \cdot Q k_{\mu}\right) Q_{\nu}-e\left(k^{2} g_{\mu \nu}-k_{\mu} k_{\nu}\right) .
$$

The second term vanishes upon contraction with $\epsilon_{\nu}(\phi)$ and we are left only with two form factors

$$
T_{\mu \nu}^{a b c}=-c\left(Q \cdot k g_{\mu \nu}-Q_{\mu} k_{\nu}\right)-e\left(k^{2} g_{\mu \nu}-k_{\mu} k_{\nu}\right) \text {. }
$$



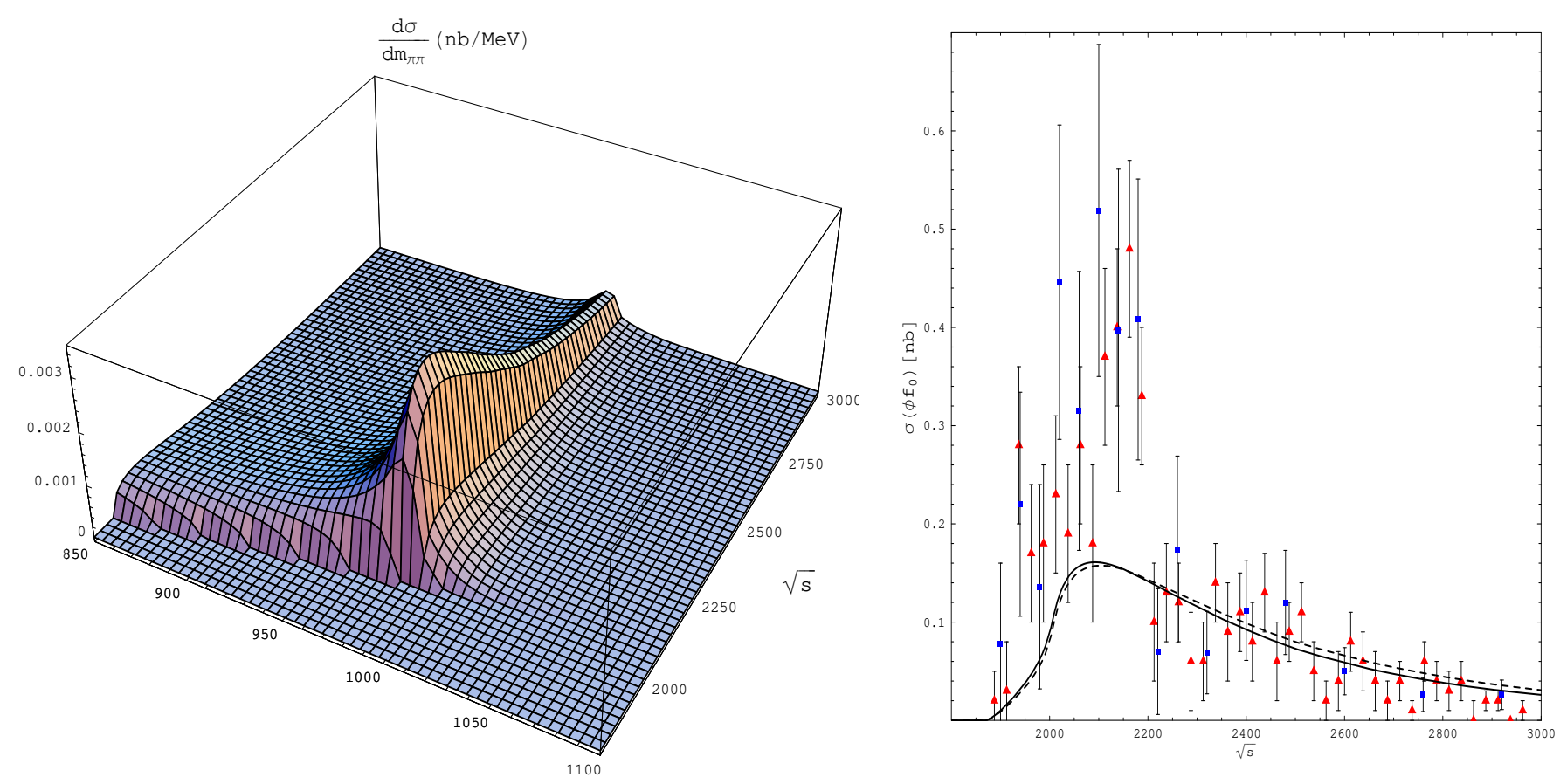

Figure 7: Left: Differential cross section as a function of the dipion invariant mass and of the center of mass energy. Right: Cross section for $e^{+} e^{-} \rightarrow \phi[\pi \pi]_{I=0}$, integrated in the $m_{\pi \pi}=850-1100 \mathrm{MeV}$ range, as a function of $\sqrt{s}$ including all contributions. Experimental points from Ref. [43], triangled (boxed) points correspond to charged (neutral) pions.

The results for the double distribution in total energy and invariant mass of two pions can be seen in fig. 7 (left), where the crest due to the $f_{0}(980)$ excitation is clearly visible. The integrated cross section around the crest of the $f_{0}(980)$, as done in the experimental analysis, is shown in fig. 7 (right). We can see there that we can reproduce the bulk of the data, in strength and shape, but the peak around 2175 $\mathrm{MeV}$ is not reproduced. There is an extra cross section with an approximate Breit Wigner shape which suggest the excitation of a resonance which decays into $\phi f_{0}(980)$. This was the conclusion of [42, 43] based on a best fit to the data. Our study offers and extra support to this claim by showing that known dynamics of the processes can explain the background, but not the peak.

\section{$6 \quad$ Electroproduction and form factors of the $N^{*}(1535)$}

We now show some new results on the form factors of the $N^{*}(1535)$ obtained from the electroproduction of this resonance, see fig. 8.

There are two independent amplitudes for the electro-transition from $J^{P}=1 / 2^{+}$to $1 / 2^{-}, A_{1 / 2}$ and $S_{1 / 2}$, which are defined in terms of the transition electric current $J_{\mu}$ by

$$
\begin{aligned}
& A_{1 / 2}=\sqrt{\frac{2 \pi \alpha}{q_{R}}} \frac{1}{e}\left\langle N^{*}, J_{z}=\frac{1}{2}\left|\epsilon_{\mu}^{(+)} J^{\mu}\right| N, S_{z}=-\frac{1}{2}\right\rangle \\
& S_{1 / 2}=\sqrt{\frac{2 \pi \alpha}{q_{R}}} \frac{1}{e} \frac{|\vec{k}|}{\sqrt{Q^{2}}}\left\langle N^{*}, J_{z}=\frac{1}{2}\left|\epsilon_{\mu}^{(0)} J^{\mu}\right| N, S_{z}=\frac{1}{2}\right\rangle
\end{aligned}
$$

with the fine structure constant $\alpha=e^{2} / 4 \pi$, the energy equivalent of a real photon $q_{R}=\left(W^{2}-M_{N}^{2}\right) /(2 W)$ and the photon-nucleon center-of-mass energy $W \equiv \sqrt{P^{2}}$. The polarization vectors of the photon, $\epsilon_{\mu}$, are given by

$$
\epsilon_{\mu}^{ \pm}=\frac{1}{\sqrt{2}}(0, \mp 1,-i, 0) ; \quad \epsilon_{\mu}^{0}=\frac{1}{\sqrt{Q^{2}}}\left(k, 0,0,-k^{0}\right)
$$




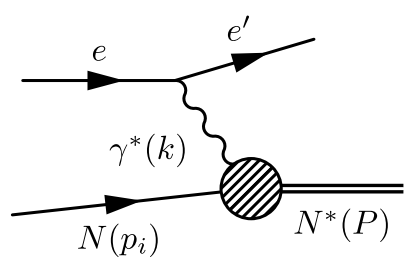

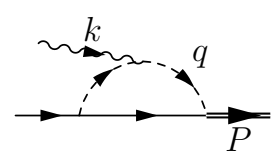

(a)

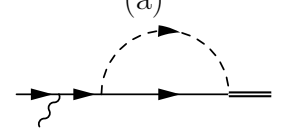

(d)

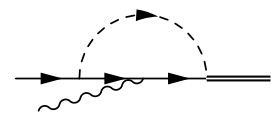

(b)

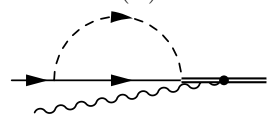

(e)

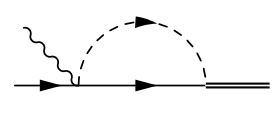

(c)

Figure 8: Letf: Kinematics of the electroproduction of $N(1535)$. Right: Feynman diagrams for the transition form factor of $N(1535)$ at one loop level. The solid, dashed, wavy and double lines denote octet baryons, mesons, photon and $N(1535)$, respectively.

with $Q^{2}=-k^{2}$, where we take the CM momenta $\vec{k}$ and $\vec{p}_{i}$ along the $z$ axis.

It can be seen, using the equation of motion for the spinors and conservation of momenta, that the transition current $J^{\mu}$ can be written, in general, by the following three Lorentz scalar amplitudes:

$$
J^{\mu}=\left(\mathcal{M}_{1} \gamma^{\mu}+\mathcal{M}_{2} P^{\mu}+\mathcal{M}_{3} k^{\mu}\right) \gamma_{5}
$$

The gauge invariance $k \cdot J=0$, tells us that there are only two independent amplitudes among these three amplitudes, $\mathcal{M}_{i}$, giving the following relation:

$$
\left(M_{N^{*}}+M_{N}\right) \mathcal{M}_{1}+k \cdot P \mathcal{M}_{2}+k^{2} \mathcal{M}_{3}=0
$$

Since the $N^{*}(1535)$ is also dynamically generated in our approach, we obtain the electroproduction amplitude by coupling the photon to the meson-baryon components that build up this resonance [11. The mechanisms are depicted in fig.8 (right), which produce a gauge invariant set of diagrams.
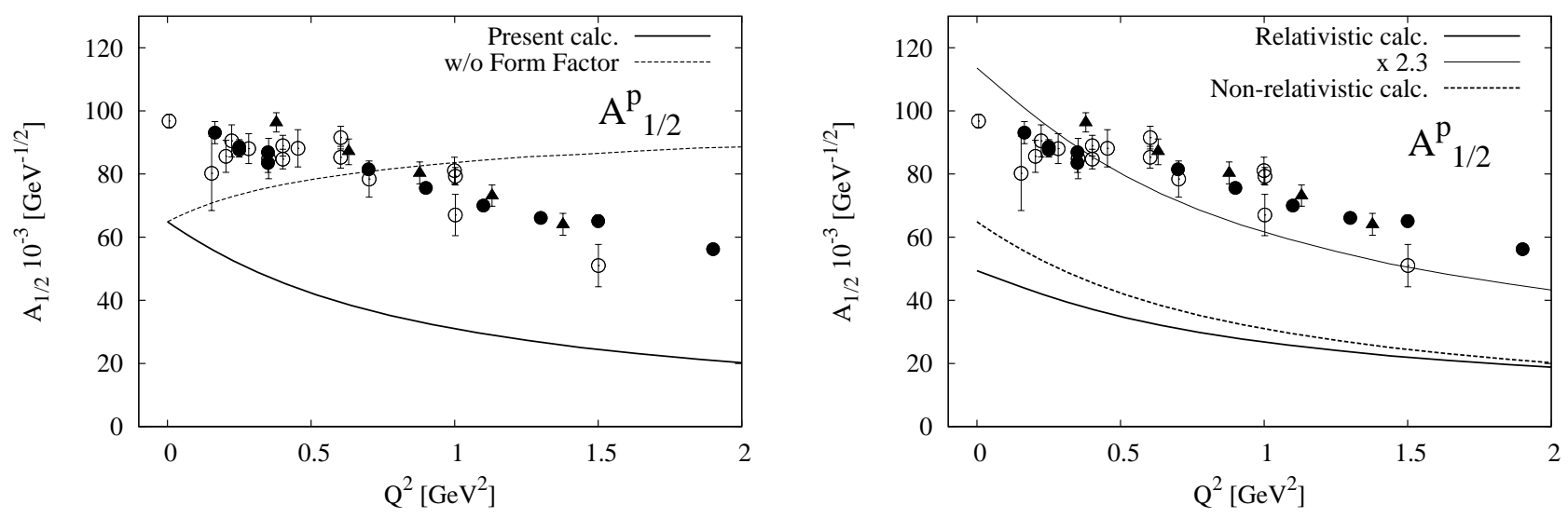

Figure 9: Left: Modulus of the $A_{1 / 2}$ helicity amplitude for the proton resonance as a function of $Q^{2}$ with $W=1535 \mathrm{MeV}$ calculated in the non-relativistic formulation. The solid (dotted) line shows the $A_{1 / 2}^{p}$ amplitude with (without) the form factor of the meson inside the loops. Marks with error bars are experimental data normalized by the $N^{*}$ full width $\Gamma_{N^{*}}=150 \mathrm{MeV}$ and the $N^{*} \rightarrow \eta N$ branching ratio $b_{\eta}=0.55$. Filled triangles and circles are results of the CLAS collaboration taken from Refs. [49] and [48], respectively. Open circles show results of Refs. [47, 50, 51, 52]. Righ: Modulus of the $A_{1 / 2}$ helicity amplitude for the proton resonance as a function of $Q^{2}$ with $W=1535 \mathrm{MeV}$ in relativistic formulation. The solid (dotted) line shows the $A_{1 / 2}^{p}$ amplitude with (without) the form factor of the meson inside the loops. 

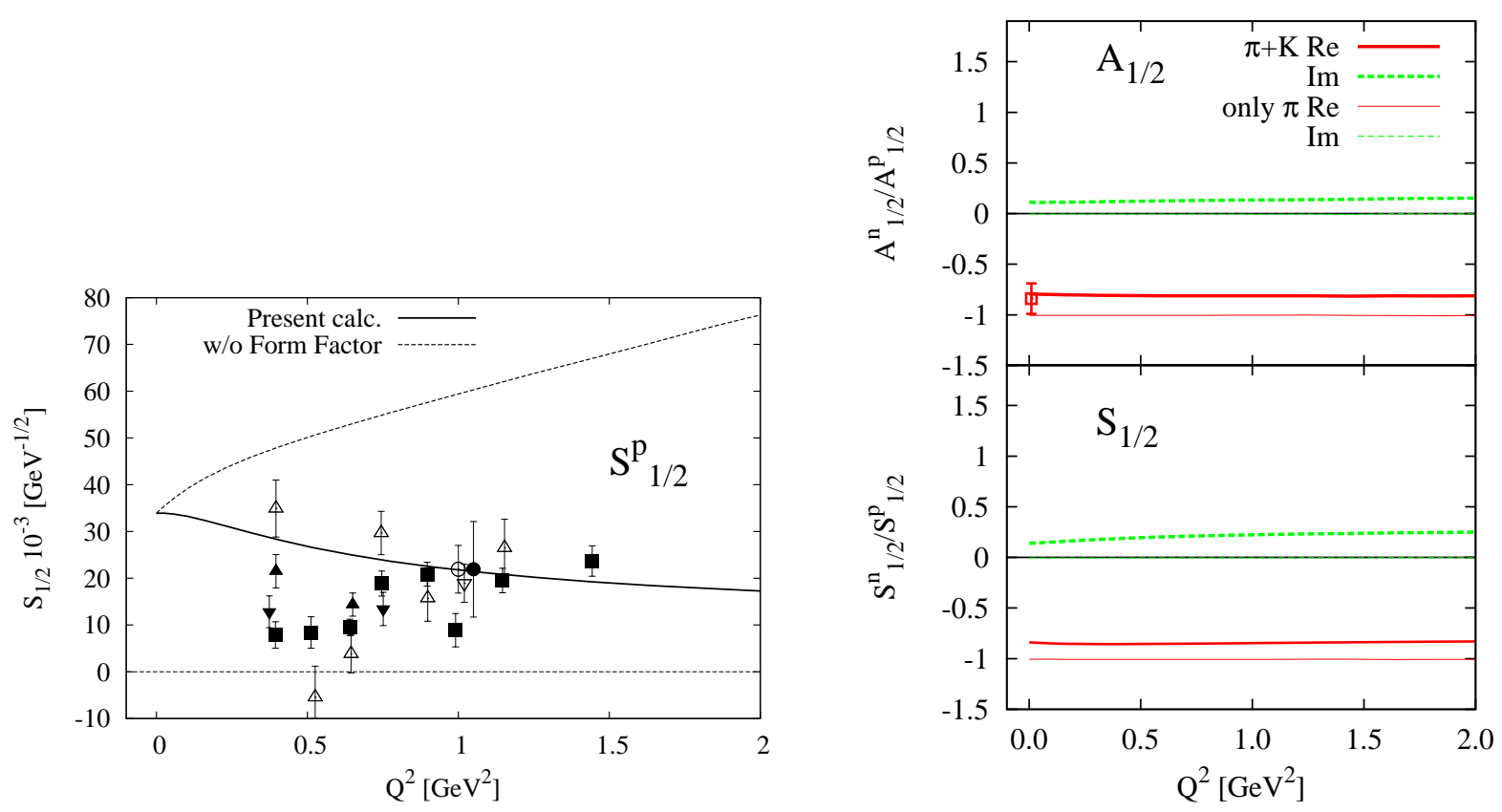

Figure 10: Left: Modulus of the $S_{1 / 2}$ helicity amplitude for the proton resonance as a function of $Q^{2}$ with $W=1535 \mathrm{MeV}$. The solid (dotted) line shows the $S_{1 / 2}^{p}$ amplitude with (without) the form factor of the meson inside the loops. The sign of this amplitude relative to $A_{1 / 2}^{p}$ is negative, both in experiment and theory. Right: The $n / p$ ratios of the helicity amplitudes with $W=1535 \mathrm{MeV}$. The upper and lower panels show the $n p$ ratios of the $A_{1 / 2}$ and $S_{1 / 2}$ amplitudes, respectively. The open square shows the value given in Ref. [53].

The results obtained can be seen in figs. 9, 10. We can see there the $A_{1 / 2}$ and $S_{1 / 2}$ form factors for the proton and neutron as a function of $Q^{2}$ compared with experiment. We have done both a relativistic and a non relativistic calculation. In both cases the results lye a bit below the data. The $Q^{2}$ dependence, when the results are scaled to the experimental values, is fair, particularly for the relativistic case, see fig. 9 (right). We should note, however, that the experimental values are obtained from measured cross sections making particular assumptions of the $N^{*}(1535)$ width and branching ratio to $\eta p$ and these uncertainties are not reflected in the data shown in the figures. It is instructive to mention that in the latest version of the MAID2007 global analysis of data [54] the value of the $A_{1 / 2}$ amplitude for real photons has the value $6610^{-3} \mathrm{GeV}^{-1 / 2}$, which is in agreement with our results.

In any case it is also interesting to observe that the ratio of neutron to proton amplitudes, which is free of the global normalization, appears in our approach in good agreement with the data.

\section{$7 \quad$ Photoproduction of $\omega$ and $\omega$ in the nuclear medium}

The interaction of vector mesons with nuclei has captured for long the attention of the hadron community. Along these lines, an approach has been followed by the CBELSA/ TAPS collaboration by looking at the $\gamma \pi^{0}$ coming from the $\omega$ decay, where a recent work [55] claims evidence for a decrease of the $\omega$ mass in the medium of the order of $100 \mathrm{MeV}$ from the study of the modification of the mass spectra in $\omega$ photoproduction. Here we present the reanalysis of the data of [55] done in [56], where one concludes that the distribution is compatible with an enlarged $\omega$ width of about $90 \mathrm{MeV}$ at nuclear matter density and no shift in the mass and at the same time we show the insensitivity of the results to a mass shift. We also show results for the $(\gamma, p)$ reaction searching for possible $\omega$ bound states in 
the nucleus concluding that even in the case of a sufficiently attractive potential and small width no peaks can be seen with the present experimental resolution of about $50 \mathrm{MeV}$ at ELSA. We also discuss the origin of a two peak structure of the $(\gamma, p)$ cross section which should not me misidentified with evidence for an $\omega$ bound state in the nucleus.

\section{Preliminaries}

We consider the photonuclear reaction $A\left(\gamma, \omega \rightarrow \pi^{0} \gamma\right) X$ in two steps - production of the $\omega$-mesons and propagation of the final states. In the laboratory, where the nucleus with the mass number $A$ is at rest, the nuclear total cross section of the inclusive reaction $A(\gamma, \omega) X$, including the effects of Fermi motion and Pauli blocking, plus effects of final state interaction of the particles produced, can be calculated as shown in [56].

The $\omega$-mesons are produced according to their spectral function $S_{\omega}$ at a local density $\rho(r)$

$$
\begin{aligned}
& S_{\omega}\left(m_{\omega}, \widetilde{m}_{\omega}, \rho\right)= \\
& -\frac{1}{\pi} \frac{\operatorname{Im} \Pi_{\omega}(\rho)}{\left(\widetilde{m}_{\omega}^{2}-m_{\omega}^{2}-\operatorname{Re} \Pi_{\omega}(\rho)\right)^{2}+\left(\operatorname{Im} \Pi_{\omega}(\rho)\right)^{2}},
\end{aligned}
$$

where $\Pi_{\omega}$ is the in-medium selfenergy of the $\omega$. The width of the $\omega$ in the nuclear medium is related to the selfenergy by $\Gamma_{\omega}\left(\rho, \widetilde{m}_{\omega}\right)=-\operatorname{Im}_{\omega}\left(\rho, \widetilde{m}_{\omega}\right) / E_{\omega}$. It includes the free width $\Gamma_{\text {free }}=8.49 \mathrm{MeV}$ and an in-medium part $\Gamma_{\text {coll }}(\rho)$ which accounts for the collisional broadening of the $\omega$ due to the quasielastic and absorption channels. In Eq. (22) $\operatorname{Re} \Pi_{\omega}=2 E_{\omega} \operatorname{Re} V_{\text {opt }}(\rho)$, where $V_{\text {opt }}(\rho)$ is the $\omega$ nucleus optical potential accounts for a possible shift of the $\omega$ mass in the medium and we shall make some considerations about it latter on.

We also consider the situation when the energy of the incident photon beam is not fixed but constrained in some energy interval $E_{\gamma}^{\min }<E_{\gamma}<E_{\gamma}^{\max }$, and also take into account the photon flux produced at the ELSA facility.

\section{The Monte Carlo simulation procedure}

The computer MC simulation proceeds in close analogy to the actual experiment. At first, the multiple integral involved in the evaluation of the cross section is carried out using the MC integration method. This procedure provides a random point $\vec{r}$ inside the nucleus where the photon collides with the nucleon, also randomly generated from the Fermi sea with $\left|\vec{p}_{N}\right| \leq k_{F}(\vec{r})$. For the sample event in the MC integral the mass $\widetilde{m}_{\omega}$ of the $\omega$ respects the spectral function $S_{\omega}$ at local density $\rho(r)$, see Eq. (22). Inside the nucleus the $\omega$-mesons moving with the three momentum $\vec{p}_{\omega}^{l a b}$ necessarily interact with the nucleons in their way out of the nucleus. In the $\mathrm{MC}$ simulation the $\omega$-mesons are allowed to propagate a distance $\delta \vec{L}=\frac{\vec{p}_{\omega}^{l a b}}{\left|\vec{p}_{\omega}^{l a b}\right|} \delta L$ and at each step, $\delta L \simeq 0.1 \mathrm{fm}$, the reaction probabilities for different channels like the decay of the $\omega$ into $\pi^{0} \gamma$ and $\pi \pi \pi$ final states, quasielastic scattering and in-medium absorption are properly calculated. Details of the simulation can be seen in [56].

We use the following parameterization for the width, $\Gamma_{a b s}=\Gamma_{0} \frac{\rho(r)}{\rho_{0}}$, where $\rho_{0}=0.16 \mathrm{fm}^{-3}$ is the normal nuclear matter density.

The propagation of pions in nuclei is done using a MonteCarlo simulation procedure. In their way out of the nucleus pions can experience the quasielastic scattering or can be absorbed. The intrinsic probabilities for these reactions as a function of the nuclear matter density are calculated using the phenomenological model of Refs [57], which also includes higher order quasielastic cuts and the twobody and three-body absorption mechanisms. Details for the present case are described in [56]. 

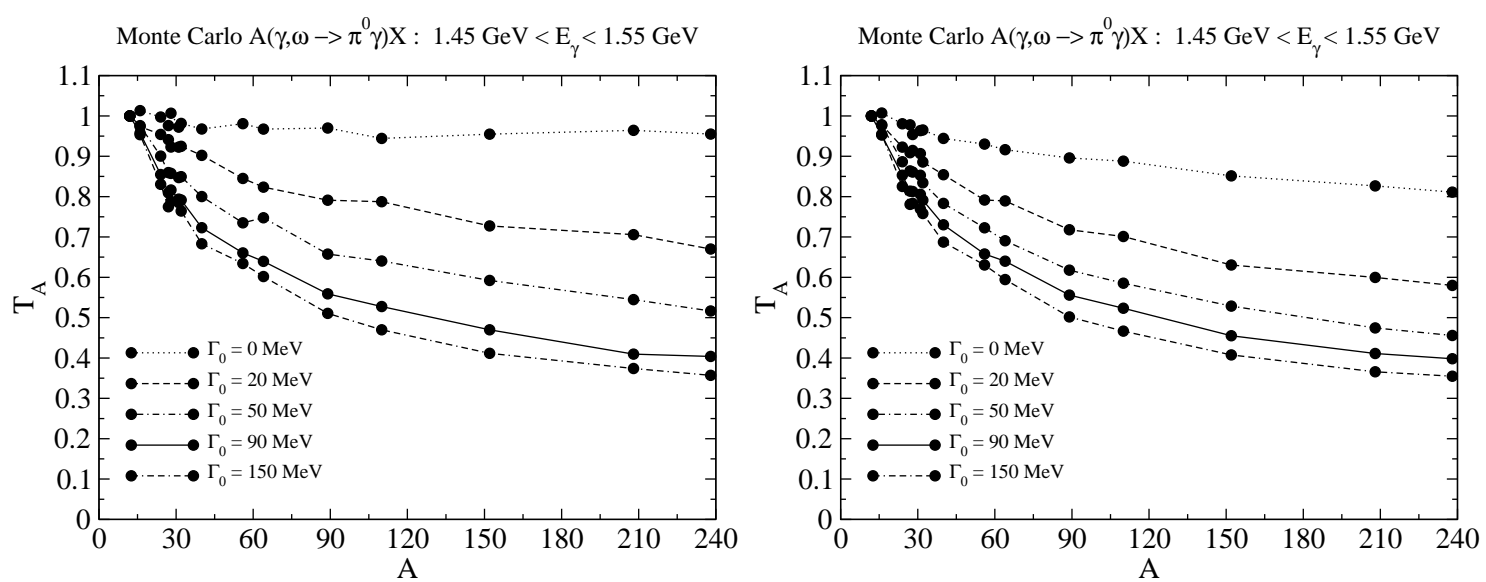

Figure 11: The result of the Monte Carlo method for the $A$-dependence of the nuclear transparency ratio $T_{A}$ without (left panel) and with (right panel) FSI of outgoing pions. A lower cut $T_{\pi}>150 \mathrm{MeV}$ on the kinetic energy of the outgoing pions has been used to suppress the contribution of the distorted events due to FSI. The incident photon beam was constrained in the range $1.45 \mathrm{GeV}<E_{\gamma}<1.55 \mathrm{GeV}$. The carbon ${ }^{12} \mathrm{C}$ was used as the reference target in the ratio of the nuclear cross sections. With $\Gamma_{a b s}=\Gamma_{0} \frac{\rho(r)}{\rho_{0}}$, where $\rho_{0}$ is the normal nuclear matter density, the dotted, dashed, dash-dotted, solid and dash-dash-dotted curves correspond to $\Gamma_{0}=0 \mathrm{MeV}, \Gamma_{0}=20 \mathrm{MeV}, \Gamma_{0}=50 \mathrm{MeV}, \Gamma_{0}=90 \mathrm{MeV}$ and $\Gamma_{0}=150 \mathrm{MeV}$, respectively.

\section{In-medium $\omega$-meson width and nuclear transparency}

In this section we discuss an extraction of the in-medium inelastic width of the $\omega$ in the photonuclear experiments. As a measure for the $\omega$-meson width in nuclei we employ the so-called nuclear transparency ratio

$$
\tilde{T}_{A}=\frac{\sigma_{\gamma A \rightarrow \omega X}}{A \sigma_{\gamma N \rightarrow \omega X}}
$$

i.e. the ratio of the nuclear $\omega$-photoproduction cross section divided by $A$ times the same quantity on

a free nucleon. $\tilde{T}_{A}$ describes the loss of flux of $\omega$-mesons in the nuclei and is related to the absorptive part of the $\omega$-nucleus optical potential and thus to the $\omega$ width in the nuclear medium.

We have done the $\mathrm{MC}$ calculations for the sample nuclear targets: ${ }_{6}^{12} \mathrm{C},{ }_{8}^{16} \mathrm{O},{ }_{12}^{24} \mathrm{Mg},{ }_{13}^{27} \mathrm{Al},{ }_{14}^{28} \mathrm{Si},{ }_{15}^{31} \mathrm{P}$, ${ }_{16}^{32} \mathrm{~S},{ }_{20}^{40} \mathrm{Ca},{ }_{26}^{56} \mathrm{Fe},{ }_{29}^{64} \mathrm{Cu},{ }_{39}^{89} \mathrm{Y},{ }_{48}^{110} \mathrm{Cd},{ }_{62}^{152} \mathrm{Sm},{ }_{82}^{208} \mathrm{~Pb},{ }_{92}^{238} \mathrm{U}$. In the following we evaluate the ratio between the nuclear cross sections in heavy nuclei and a light one, for instance ${ }^{12} \mathrm{C}$, since in this way, many other nuclear effects not related to the absorption of the $\omega$ cancel in the ratio, $T_{A}$.

The results of the $\mathrm{MC}$ calculation for the $A$-dependence of the nuclear transparency ratio $T_{A}$ are presented in Fig. 11. The incident photon beam was constrained in the range $1.45 \mathrm{GeV}<E_{\gamma}<1.55 \mathrm{GeV}$ - a region which is considered in the analysis of the CBELSA/TAPS experiment [58, 59]. In Fig. 11 (left panel) we show the results for the transparency ratio when the collisional broadening and FSI of the $\omega$ are taken into account but without FSI of the pions from $\omega \rightarrow \pi^{0} \gamma$ decays inside the nucleus. The right panel corresponds to considering in addition the FSI of the pions.

By using these results and taking into account the preliminary results of CBELSA/TAPS experiment [59] we get an estimate for the $\omega$ width $\Gamma_{a b s} \simeq 90 \times \frac{\rho(r)}{\rho_{0}} \mathrm{MeV}$. This estimate must be understood as an average over the $\omega$ three momentum.

\section{In-medium $\omega$-meson mass and CBELSA/TAPS experiment}

The first thing one should note is that the $\omega$ line shape reconstructed from $\pi^{0} \gamma$ events strongly depends on the background shape subtracted from the bare $\pi^{0} \gamma$ signal. In Ref. [55] the shape of the background 

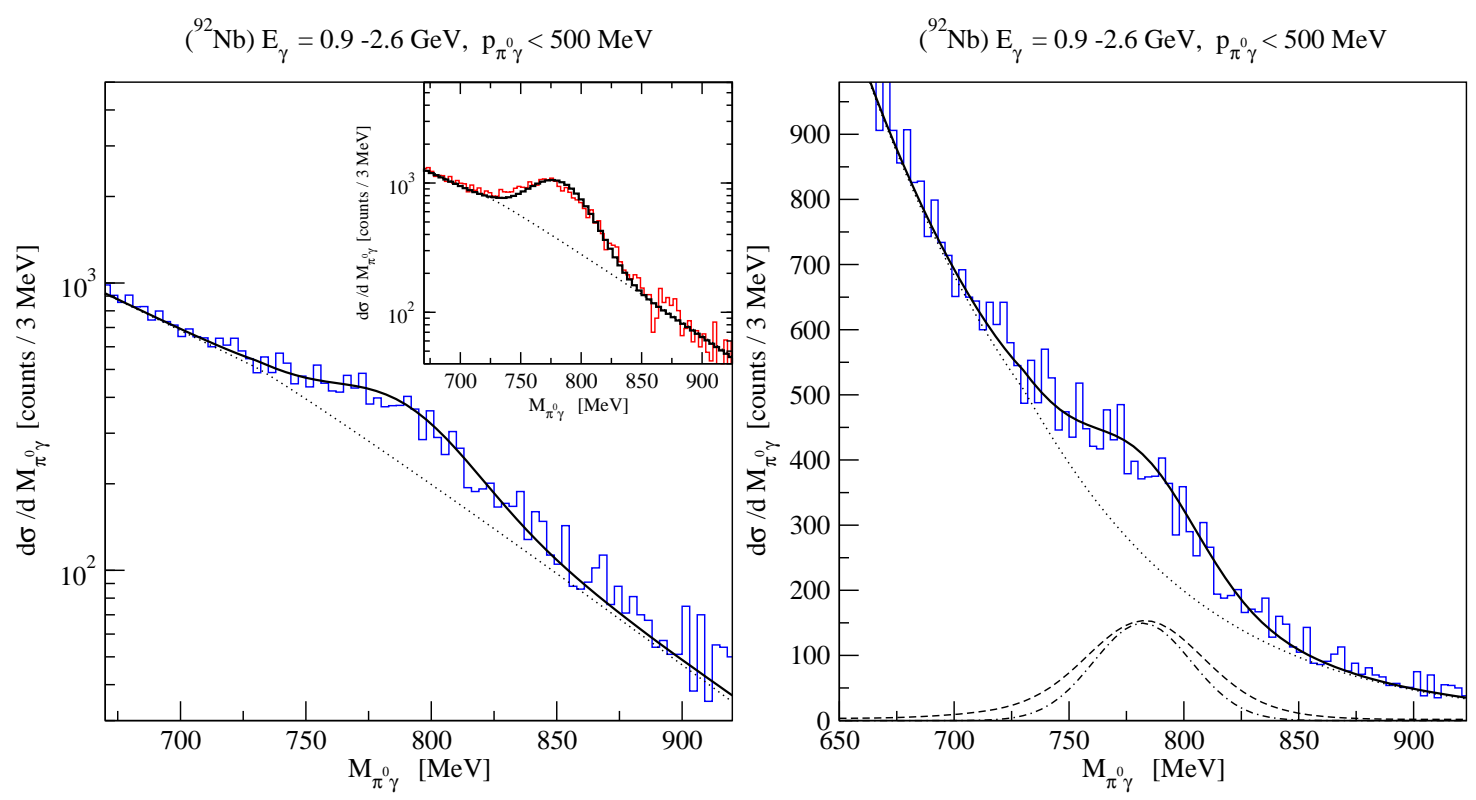

Figure 12: Left panel: Invariant mass spectra reconstructed from the $\pi^{0} \gamma$ events in the $\left(\gamma, \pi^{0} \gamma\right)$ reaction from Nb target (solid curve). The experimental data are from Ref. [55. The incident photon beam has been constrained in the range $0.9 \mathrm{GeV}<E_{\gamma}^{i n}<2.6 \mathrm{GeV}$. Dotted curve is an uncorrelated $\pi^{0} \gamma$ background (see the text). Right panel: Same but in linear scale. The dashed and dash-dash-dotted curves correspond to the $\omega \rightarrow \pi^{0} \gamma$ events with and without the kinematic cut $\left|\vec{p}_{\pi^{0} \gamma}\right|<500 \mathrm{MeV}$, respectively. The normalization without cut is arbitrary. The solid line corresponds to the sum of the background and the dashed line. Inset (left panel): The $\pi^{0} \gamma$ invariant mass spectra in the elementary $p\left(\gamma, \pi^{0} \gamma\right) p$ reaction. Same background line shape (dotted curve) as for the Nb target has been used. The solid line is the sum of the background and $\omega \rightarrow \pi^{0} \gamma$ events.

was chosen such that it accounted for all the experimental strength at large invariant masses. This choice was done both for the elementary $\gamma p \rightarrow \pi^{0} \gamma p$ reaction as well as for nuclei. As we shall show, this choice of background in nuclei implies a change of the shape from the elementary reaction to that in the nucleus for which no justification was given. We shall also show that when the same shape for the background as for the elementary reaction is chosen, the experiment in nuclei shows strength at invariant masses higher than $m_{\omega}$ where the choice of [55] necessarily produced no strength. We will also see that the experimental data can be naturally interpreted in terms of the large in-medium $\omega$ width discussed above without the need to invoke a shift in the $\omega$ mass in the medium.

In Fig. 12 we show the experimental data (solid histogram) for the $\pi^{0} \gamma$ invariant mass spectra in the reaction $\left(\gamma, \pi^{0} \gamma\right)$ [55] from ${ }_{41}^{92} \mathrm{Nb}$ target. The inset (left panel) corresponds to the $\pi^{0} \gamma$ spectra from the hydrogen target. In our MC calculations the incident photon beam has been constrained in the range $0.9 \mathrm{GeV}<E_{\gamma}^{i n}<2.6 \mathrm{GeV}$. The higher momentum cut $\left|\vec{p}_{\pi^{0} \gamma}\right|=\left|\vec{p}_{\pi^{0}}+\vec{p}_{\gamma}\right|<500 \mathrm{MeV}$ on a three momentum of the $\pi^{0} \gamma$ pair was imposed as in the actual experiment. First, we use the hydrogen target, see inset in Fig. 12 (left panel), to fix the contribution of the uncorrelated $\pi^{0} \gamma$ background (dotted curve) which together with the $\pi^{0} \gamma$ signal from $\omega \rightarrow \pi^{0} \gamma$ decay, folded with the Gaussian experimental resolution of $55 \mathrm{MeV}$ as in Ref. [55], gives a fair reproduction of the experimental spectra. Then we assume the same shape of the $\pi^{0} \gamma$ background in the photonuclear reaction. The weak effect of the FSI of the pions found in the calculation, with the cuts imposed in the experiment, strongly supports this assumption.

In the following we use the $\omega$ inelastic width of $\Gamma_{0}=90 \mathrm{MeV}$ at $\rho_{0}$. The exclusive $\omega \rightarrow \pi^{0} \gamma \mathrm{MC}$ spectra is shown by the dashed curve (right panel). The solid curve is the reconstructed $\pi^{0} \gamma$ signal after applying the cut on $\pi^{0} \gamma$ momenta and adding the background fixed when using the hydrogen target (dotted curve). Note that the shape of the exclusive $\pi^{0} \gamma$ signal without applying a cut on $\pi^{0} \gamma$ 
momenta (dash-dotted curve) is dominated by the experimental resolution and no broadening of the $\omega$ is observed. This is in agreement with data of Ref. [55]. But applying the cut one increases the fraction of in-medium decays coming from the interior of the nucleus where the spectral function is rather broad and as a result the broadening of the $\pi^{0} \gamma$ signal with respect to the signal (without cut) can be well seen. The resulting MC spectra (solid curve) shows the accumulation of the $\pi^{0} \gamma$ events from the left

and right sides of the mass spectra, and it is consistent both with our choice of the uncorrelated $\pi^{0} \gamma$ background and experimental data.

We have also done the exercise of seeing the sensitivity of the results to changes in the mass. As shown in [56], a band corresponding to having the $\omega$ mass in between $m_{\omega} \pm 40 \rho / \rho_{0} \mathrm{MeV}$ is far narrower than the statistical fluctuations. In other words, this experiment is too insensitive to changes in the mass to be used for a precise determination of the shift of the $\omega$-mass in the nuclear medium. 8 We should also note that the peak position barely moves since it is dominated by the decay of the $\omega$ outside the nucleus.

\section{Production of bound $\omega$ states in the $(\gamma, p)$ reaction}

Here we evaluate the formation rate of $\omega$ bound states in the nucleus by means of the $(\gamma, \mathrm{p})$ reaction. We use the Green function method [60] to calculate the cross sections for $\omega$-mesic states formation as described in Refs. 61] in detail. The theoretical model used here is exactly same as that used in these references.

The $\omega$-nucleus optical potential is written here as $V(r)=\left(V_{0}+i W_{0}\right) \frac{\rho(r)}{\rho_{0}}$, where $\rho(r)$ is the nuclear experimental density for which we take the two parameter Fermi distribution. We consider three cases of the potential strength as: $\left(V_{0}, W_{0}\right)=-(0,50),-(100,50) \mathrm{MeV}$ and $-(156,29) \mathrm{MeV}$. The last of the potentials is obtained by the linear density approximation with the scattering length $a=1.6+0.3 i$ $\mathrm{fm}$ [62]. This potential is strongly attractive with weak absorption and hence should be the ideal case for the formation of $\omega$ mesic nuclei. No $\omega$ bound states are expected for the first potential which has only an absorptive part. The second potential has a strong attraction with the large absorptive part as indicated in Ref. [58]. For the first two potentials we find no visible peaks in the spectrum since the width is so large. For the third potential we observe peaks but they are washed out when folded with the experimental resolution of about $50 \mathrm{MeV}$ of ELSA.

\section{Monte Carlo simulation of the reaction of the $(\gamma, p)$ reac- tion}

We next apply the MonteCarlo simulation explained above to describe the $(\gamma, p)$ reaction studied at ELSA. Because our MC calculations represent complete event simulations it is possible to take into account the actual experimental acceptance of ELSA [58] (see details in [63]).

We start our MC analysis with the cross section of the elementary reaction $\gamma p \rightarrow \omega p \rightarrow \pi^{0} \gamma p$. With this we determine the cross section for $\omega$ formation and follow the fate of the protons at the same time.

There are also sources of background like from $\gamma p \rightarrow \pi^{0} \pi^{0} p$, or $\gamma p \rightarrow \pi^{0} \eta p$, where one of the two photons from the decay of the $\pi^{0}$ or the $\eta$ is not measured. We show in Fig. 13 the cross section $d \sigma / d E_{\pi^{0} \gamma}$ coming from the $\gamma p \rightarrow \pi^{0} \pi^{0} p$ reaction followed by the decay $\pi^{0} \rightarrow \gamma \gamma$ of either of the $\pi^{0}$ (left panel) and from the $\gamma p \rightarrow \pi^{0} \eta p$ reaction followed by the decay $\eta \rightarrow \gamma \gamma$ (right panel). As one can see, the contribution from the $\pi^{0} \pi^{0}$ photoproduction to the background is the dominant one among the two. The important thing, thus, is that these two sources of background, with the cuts imposed, produce a background peaked at $-100 \mathrm{MeV}$. For the exclusive $\pi^{0} \gamma$ events coming from $\gamma p \rightarrow \omega p \rightarrow \pi^{0} \gamma p$ an experimental resolution of $50 \mathrm{MeV}$ was imposed, see Ref. [55]. We obtain a factor of two bigger 


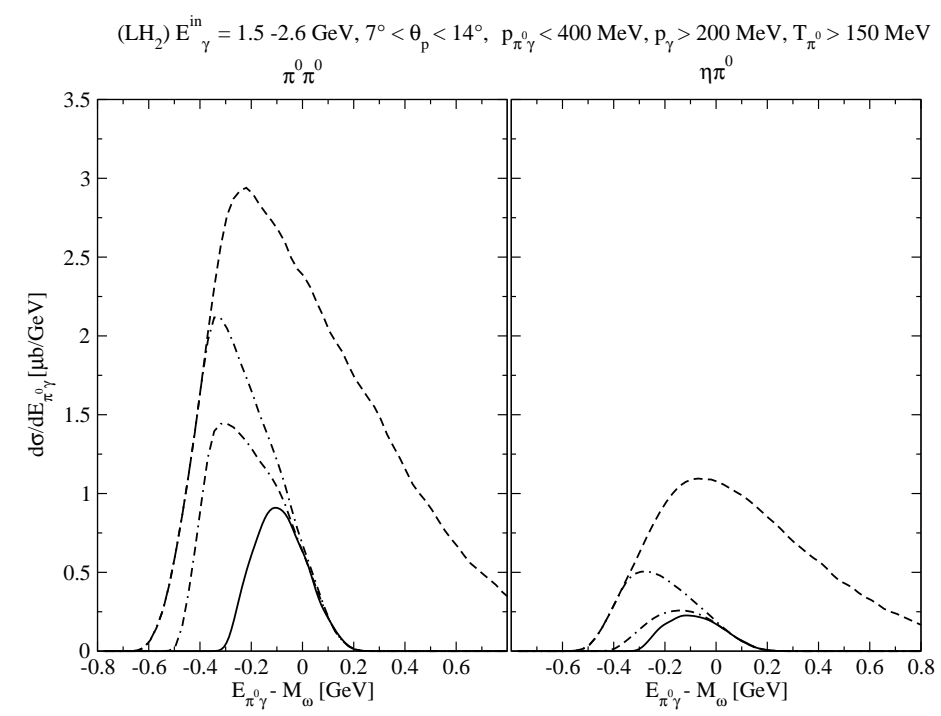

Figure 13: The differential cross section $d \sigma / d E_{\pi^{0} \gamma}$ of the reactions $\gamma p \rightarrow \pi^{0} \pi^{0} p$ (left panel) and $\gamma p \rightarrow \pi^{0} \eta p$ (right panel) followed by the decay $\pi^{0}(\eta) \rightarrow \gamma \gamma$ as a function of the $E_{\pi^{0} \gamma}-m_{\omega}$ where $E_{\pi^{0} \gamma}=E_{\pi^{0}}+E_{\gamma}$. The following cuts were imposed: $E_{\gamma}^{\text {in }}=1.5 \div 2.6 \mathrm{GeV}$ and $7^{\circ}<\theta_{p}<14^{\circ}$ (dashed curves); $E_{\gamma}^{i n}=1.5 \div 2.6 \mathrm{GeV}$, $7^{\circ}<\theta_{p}<14^{\circ}$ and $\left|\vec{p}_{\pi^{0}}+\vec{p}_{\gamma}\right|<400 \mathrm{MeV}$ (dash-dotted curves); plus the cut $T_{\pi^{0}}>150 \mathrm{MeV}$ (dash-dash-dotted curves) and plus the cut $\left|\vec{p}_{\gamma}\right|>200 \mathrm{MeV}$ (solid curves).

strength at the $\omega$ peak than at the peak from the $\gamma p \rightarrow \pi^{0} \pi^{0} p$ background. Experimentally, this seems to be also the case from the preliminary data of CBELSA/TAPS,

In the following we assume that the inclusive $\pi^{0} \gamma$ background scales with respect to the target nucleus mass number $A$ like $\sigma_{A} \simeq A \sigma_{\text {elem }}$. But this is not the case for the exclusive $\pi^{0} \gamma$ events coming from the decay of the $\omega \rightarrow \pi^{0} \gamma$, since the rather strong absorption of the $\omega$ inside the nucleus changes the scaling relation and $\sigma_{A}\left(\omega \rightarrow \pi^{0} \gamma\right) \simeq A^{\alpha} \sigma_{\text {elem }}\left(\omega \rightarrow \pi^{0} \gamma\right)$, where the attenuation parameter $\alpha<1$.

In Fig. 14 we show the result of the MC simulation for the $E_{\pi^{0} \gamma}-m_{\omega}$ spectra reconstructed from the $\pi^{0}$ and $\gamma$ events. The calculations are performed for the sample nuclear targets ${ }^{12} \mathrm{C},{ }^{40} \mathrm{Ca},{ }^{92} \mathrm{Nb}$ and ${ }^{208} \mathrm{~Pb}$. The kinematic and acceptance cuts discussed before have been already imposed. The MC distributions are normalized to the nuclear mass number $A$. The solid curves correspond to the sum of the inclusive $\pi^{0} \gamma$ background (dash-dotted curve), and the exclusive $\pi^{0} \gamma$ events coming from the direct decay of the $\omega \rightarrow \pi^{0} \gamma$. The contributions of the exclusive $\omega \rightarrow \pi^{0} \gamma$ events are shown by the dashed curves. We note a very strong attenuation of the $\omega \rightarrow \pi^{0} \gamma$ signal with respect to the background contribution with increasing nuclear mass number $A$. This is primary due to the stronger absorption of the $\omega$-mesons with increasing nuclear matter density. The former exercise indicates that given the particular combination of $\pi^{0} \gamma$ from an uncorrelated background and from $\omega$ decay, and the different behaviour of these two sources in the $\pi^{0} \gamma$ production in nuclei, a double hump structure is unavoidable in nuclei with this set up, and one should avoid any temptation to associate the lower energy peak to a possible bound state in the nucleus.

\section{Conclusions}

In this lecture we have addressed several recent topics on photoproduction of mesons on nucleons and nuclei. One of the topics was the combined study of the $\gamma p \rightarrow \pi^{0} \eta p$ and $\gamma p \rightarrow \pi^{0} K^{0} \Sigma^{+}$reactions, which, together with related pion induced reactions, showed a dominance of the $\Delta(1700)$ excitation. This resonance is one of the dynamically generated resonances in the chiral unitary approach and this 


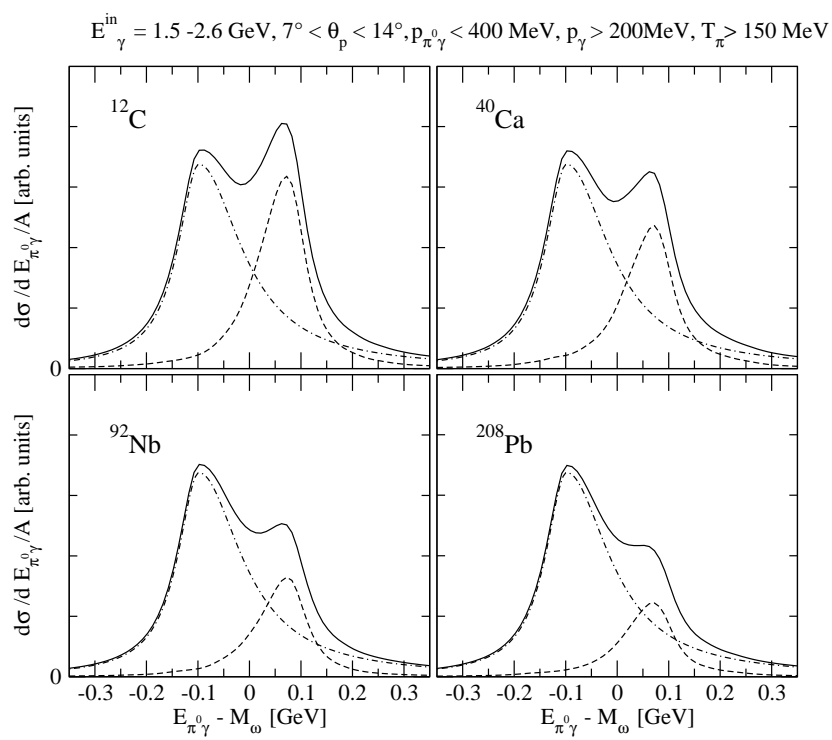

Figure 14: The differential cross section $d \sigma / d E_{\pi^{0} \gamma}$ of the reaction $A\left(\gamma, \pi^{0} \gamma\right) X$ as a function of $E_{\pi^{0} \gamma}-m_{\omega}$ from ${ }^{12} \mathrm{C},{ }^{40} \mathrm{Ca},{ }^{92} \mathrm{Nb}$ and ${ }^{208} \mathrm{~Pb}$ nuclear targets. The reconstructed exclusive events from the $\omega \rightarrow \pi^{0} \gamma$ decay are shown by the dashed curves. The $\pi^{0} \gamma$ background is shown by the dash-dotted curves. The sum of the two contributions is given by the solid curves. The following cuts were imposed: $E_{\gamma}^{\text {in }}=1.5 \div 2.6 \mathrm{GeV}, 7^{\circ}<\theta_{p}<14^{\circ}$, $\left|\vec{p}_{\pi^{0}}+\vec{p}_{\gamma}\right|<400 \mathrm{MeV},\left|\vec{p}_{\gamma}\right|>200 \mathrm{MeV}$ and $T_{\pi}>150 \mathrm{MeV}$. The exclusive $\omega \rightarrow \pi^{0} \gamma$ signal has been folded with the $50 \mathrm{MeV}$ experimental resolution. All spectra are normalized to the corresponding nuclear mass numbers $A$.

theory provides couplings unaccessible through known decay widths which allow a global understanding of these reactions.

The study of the $e^{+} e^{-} \rightarrow \phi f_{0}(980)$ reaction, using again techniques of chiral unitary theory, allowed us to calculate the bulk of the cross section for this reaction, which was well reproduced, and comparison with data showed the need to introduce a new resonance, the $\mathrm{X}(2175)$, which has been claimed from the analysis of a recent BABAR experiment.

Similarly, using information obtained in the chiral approach to the $N^{*}(1535)$, we showed results obtained for the electromagnetic form factors of this resonance, which provide a fair reproduction of the $Q^{2}$ dependence, and a normalization for real photons in agreement with the MAID2007 analysis, though a little lower than experiments without counting the systematic experimental uncertainties in the normalization.

Concerning photoproduction $\omega$, the studies done in [56] and [63] show that: 1) The ELSA results on inclusive $\omega$ production in nuclei can be interpreted in terms of a large $\omega$ width in the medium without the need of a mass shift. 2) The results are very insensitive to a mass shift in matter. 3) With the large medium $\omega$ width derived from the ELSA data no visible peaks for $\omega$ bound states are seen, even with hypothetical large $\omega$ binding. 4) Even in the hypothetical case of small widths, the possible $\omega$ bound states would not be resolved with the present ELSA resolution. 5) When looking at the $(\gamma, p)$ reaction with the present ELSA experimental set up, a double hump structure appears in the calculation from the interplay of the $\omega$ signal and the background. The peak at lower energies is related to the background, with the cuts imposed, and should not me misidentified with a possible $\omega$ bound state in the nucleus.

\section{Note added in Proofs:}

After thorough discussions with V. Metag and M. Kotulla it became clear that the issue of using the 
mixed event technique (MET) to get the background in $\omega$ production in proton and nuclear targets is very delicate. Questions like normalizations, effects of cuts, comparison of the MET results on proton and nuclear targets, and particularly, the ability of the MET to generate the background in a very correlated system like in the present case, are far from settled. In this respect, the results on the MET reported in the contribution of V. Metag in this School [arxiv 0711.4709, nucl-ex] should be taken as very preliminary, and as such, one should abstain from drawing conclusions from them.

Another topic that requires clarification is the comment in the same contribution which states " since the experimental data clearly show that the background distributions on the proton and in nuclear targets are different, the assumption made in section 11 of the present paper (and references quoted there) of a background in the nucleus proportional to the one of the proton can only lead to wrong conclusions". This statement is incorrect for several reasons: 1) The experimental data quoted above are for total cross sections and do not tell us which is the background. 2) In the work reported in section 11 of the present paper one does not claim that the background in the nucleus is proportional to the one of the proton. It shows it as a possible scenario and discusses what would happen in such a case. 3) Although we do not necessarily advocate this latter scenario, it is impossible to exclude it from the present data alone, since in ref. [56] it was proved that it is indeed one of the possibilities for a certain $\omega$ signal in the nucleus.

\section{Acknowledgments}

This work is partly supported by DGICYT contract number FIS2006-003438, the Generalitat Valenciana, the projects FPA2004-05616 (DGICYT) and SA016A07 (Junta de Castilla y Leon). The work of M.N. and C.A.V.A. was supported by CONACyT- Mexico under project 50471-F. This research is part of the EU Integrated Infrastructure Initiative Hadron Physics Project under contract number RII3-CT-2004-506078.

\section{References}

[1] J. Gasser and H. Leutwyler, Nucl. Phys. B250 (1985) 465, 517, 539.

[2] U. G. Meissner, Rep. Prog. Phys. 56 (1993) 903; V. Bernard, N. Kaiser and U. G. Meissner, Int. J. Mod. Phys. E4 (1995) 193.

[3] G. Ecker, Prog. Part. Nucl. Phys. 35 (1995) 1.

[4] E. A. Veit, B. K. Jennings, A. W. Thomas and R. C. Barrett, Phys. Rev. D 31 (1985) 1033.

[5] N. Kaiser, P. B. Siegel and W. Weise, Nucl. Phys. A 594 (1995) 325

[6] E. Oset and A. Ramos, Nucl. Phys. A635 (1998) 99.

[7] J. A. Oller and U. G. Meissner, Phys. Lett. B 500 (2001) 263

[8] J. A. Oller and E. Oset, Phys. Rev. D60 (1999) 074023.

[9] N. Kaiser, P. B. Siegel and W. Weise, Phys. Lett. B 362 (1995) 23

[10] J. Nieves and E. Ruiz Arriola, Phys. Rev. D 64 (2001) 116008

[11] T. Inoue, E. Oset and M. J. Vicente Vacas, Phys. Rev. C 65 (2002) 035204

[12] J. A. Oller, E. Oset and A. Ramos, Prog. Part. Nucl. Phys. 45 (2000) 157.

[13] C. Garcia-Recio, M. F. M. Lutz and J. Nieves, Phys. Lett. B 582 (2004) 49

[14] C. Garcia-Recio, J. Nieves and L. L. Salcedo, Phys. Rev. D 74 (2006) 034025

[15] T. Hyodo, S. I. Nam, D. Jido and A. Hosaka, Phys. Rev. C 68 (2003) 018201

[16] T. Hyodo, D. Jido and A. Hosaka, Phys. Rev. Lett. 97 (2006) 192002 
[17] E. Oset, A. Ramos and C. Bennhold, Phys. Lett. B 527 (2002) 99 [Erratum-ibid. B 530 (2002) 260].

[18] D. Jido, J.A. Oller, E. Oset, A. Ramos, U.G. Meissner, Nucl. Phys. A 725 (2003) 181.

[19] E. Oset, D. Cabrera, V. K. Magas, L. Roca, S. Sarkar, M. J. Vicente Vacas and A. Ramos, Pramana 66 (2006) 731

[20] E. E. Kolomeitsev and M. F. M. Lutz, Phys. Lett. B 585 (2004) 243

[21] S. Sarkar, E. Oset and M. J. Vicente Vacas, Nucl. Phys. A 750 (2005) 294 [Erratum-ibid. A 780 (2006) 78]

[22] E. Jenkins and A. V. Manohar, Phys. Lett. B 259 (1991) 353.

[23] Pions and Nuclei, T. E. O. Ericson and W. Weise, Oxford Science Publications, 1988.

[24] T. R. Hemmert, B. R. Holstein and J. Kambor, J. Phys. G 24 (1998) 1831

[25] M. Savage, http://www.phys.washington.edu/ savage/

[26] M. F. M. Lutz and E. E. Kolomeitsev, Nucl. Phys. A 700 (2002) 193

[27] L. Roca, S. Sarkar, V. K. Magas and E. Oset, Phys. Rev. C 73 (2006) 045208

[28] M. Doring, E. Oset and D. Strottman, Phys. Rev. C 73 (2006) 045209

[29] M. Doring, E. Oset and D. Strottman, Phys. Lett. B 639 (2006) 59

[30] O. I. Dahl, L. M. Hardy, R. I. Hess et.al., Phys. Rev. 163, 1337 (1967).

[31] L. J. Curtis, C. T. Coffin, D. I. Meyer, and K. M. Terwilliger, Phys. Rev. 132, 1771 (1963).

[32] P. Hanson, G. E. Kalmus and J. Louie, Phys. Rev. D 4, 1296 (1971).

[33] D. Grether, G. Gidal and G. Borreani, Phys. Rev. D 7, 3200 (1973).

[34] D. W. Thomas, A. Engler, H. E. Fisk and R. W. Kraemer, Nucl. Phys. B 56, 15 (1973).

[35] F. Wieland, private communication.

[36] M. Nanova at the "International Workshop On The Physics Of Excited Baryons (NSTAR 05)", 10-15 Oct 2005, Tallahassee, Florida

[37] T. Nakabayashi et al., Phys. Rev. C 74 (2006) 035202.

[38] J. Ajaka et al., preprint 2007. Phys. Rev. Lett. in print.

[39] R. Erbe et al. [Aachen-Berlin-Bonn-Hamburg-Heidelberg-Muenchen Collaboration], Phys. Rev. 188, 2060 (1969).

[40] R. Erbe et al. [Aachen-Berlin-Bonn-Hamburg-Heidelberg-Muenchen Collaboration], Nuovo Cimento 49A, 504 (1967).

[41] Cambridge Bubble Chamber Group, Phys. Rev. 156, 1426 (1966)

[42] B. Aubert et al. [BABAR Collaboration], Phys. Rev. Lett. 95, 142001 (2005)

[43] B. Aubert et al. [BABAR Collaboration], Phys. Rev. D 74, 091103 (2006) ; B. Aubert et al. [BABAR Collaboration], Phys. Rev. D 76 (2007) 012008 [arXiv:0704.0630 [hep-ex]].

[44] E. Marco, S. Hirenzaki, E. Oset and H. Toki, Phys. Lett. B 470, 20 (1999) ; J. E. Palomar, L. Roca, E. Oset and M. J. Vicente Vacas, Nucl. Phys. A 729, 743 (2003) ;

[45] V. E. Markushin, Eur. Phys. J. A 8, 389 (2000); J. A. Oller, Nucl. Phys. A 714, 161 (2003)

[46] M. Napsuciale, E. Oset, K. Sasaki and C. A. Vaquera-Araujo, Phys. Rev. D 76 (2007) 074012

[47] B. Krusche et al., Phys. Rev. Lett. 74 (1995) 3736.

[48] H. Denizli et al. [CLAS Collaboration], Phys. Rev. C 76, 015204 (2007)

[49] R. Thompson et al. [CLAS Collaboration], Phys. Rev. Lett. 86, 1702 (2001)

[50] F. W. Brasse et al., Nucl. Phys. B 139, 37 (1978); F. W. Brasse et al., Z. Phys. C 22, 33 (1984). 
[51] U. Beck et al., Phys. Lett. B 51, 103 (1974).

[52] H. Breuker et al., Phys. Lett. B 74, 409 (1978).

[53] N. C. Mukhopadhyay, J. F. Zhang and M. Benmerrouche, Phys. Lett. B 364, 1 (1995)

[54] D. Drechsel, S. S. Kamalov and L. Tiator, arXiv:0710.0306 [nucl-th].

[55] D. Trnka et al. [CBELSA/TAPS Collaboration], Phys. Rev. Lett. 94 (2005) 192303

[56] M. Kaskulov, E. Hernandez and E. Oset, Eur. Phys. J. A 31 (2007) 245

[57] L. L. Salcedo, E. Oset, M. J. Vicente-Vacas and C. Garcia-Recio, Nucl. Phys. A 484 (1988) 557.

[58] D. Trnka, PhD Thesis, University of Giessen, 2006.

[59] M. Kotulla, arXiv:nucl-ex/0609012.

[60] O. Morimatsu, K. Yazaki, Nucl. Phys. A 435 (1985) 727;

[61] D. Jido, H. Nagahiro, S. Hirenzaki, Phys. Rev. C 66 (2002) 045202;

[62] F. Klingl, T. Waas and W. Weise, Nucl. Phys. A 650 (1999) 299

[63] M. Kaskulov, H. Nagahiro, S. Hirenzaki and E. Oset, Phys. Rev. C75 (2007) 064616 